\title{
Concentração plasmática de diclofenaco sódico em cães, submetidos à fonoforese
}

\author{
[Plasma concentration of diclofenac sodium in dogs submitted to diclofenac phonophoresis] \\ C. Pelizzari ${ }^{1}$, A.G. Raiser ${ }^{1}$, A. Mazzanti ${ }^{1}$, S.F. Souza ${ }^{2}$, V.M.V. Martins ${ }^{3}$, \\ R.G. Peccinini ${ }^{4}$, M.L. Campos $^{4}$ \\ ${ }^{1}$ Aluno de pós-graduação - Universidade Federal de Santa Maria - UFSM - Santa Maria, RS \\ ${ }^{2}$ Universidade Federal do Acre - UFAC - Rio Branco, AC \\ ${ }^{3}$ Universidade do Estado de Santa Catarina - UDESC - Lages, SC \\ ${ }^{4}$ Universidade Estadual Júlio de Mesquita Filho - UNESP - Araraquara, SP \\ RESUMO
}

\begin{abstract}
Com o objetivo de avaliar a concentração plasmática de diclofenaco sódico (DS) emulgel em cães com ou sem o uso de fonoforese e de verificar se a fonoforese induz à maior absorção desse fármaco, foram utilizados cinco cães, e todos eles passaram por oito grupos distintos. Um grupo recebeu, via oral, um comprimido de DS, 40mg, por animal, e sete grupos receberam aplicação transdérmica de diclofenaco sódico emulgel por ultrassom. Pela via transdérmica, a área de aplicação era de $20 \mathrm{~cm}^{2}$. A frequência do ultrassom foi de $1 \mathrm{MHz}$, modo contínuo, com intensidade de $0,4 \mathrm{Wcm}^{-2}$. Colheram-se amostras de sangue antes de se executarem os protocolos - tempo zero -, após uma hora - tempo 1 - e após quatro horas da aplicação - tempo 2 - em todos os grupos, e realizou-se análise das amostras por cromatografia líquida de alta eficiência. Houve diferença $(\mathrm{P}<0,05)$ apenas nas amostras no tempo 1 do grupo que recebeu dose oral de DS em relação às outras amostras. Não foi possível verificar concentração plasmática de diclofenaco sódico com aplicação tópica em cães submetidos ou não à fonoforese, apenas quantificou-se o diclofenaco sódico pela administração via oral. A facilitação da penetração transdérmica pelo ultrassom não foi verificada sob o protocolo especificado nesta pesquisa.
\end{abstract}

Palavras-chave: cão, sonoforese, diclofenaco sódico, CLAE

\begin{abstract}
The aim of this study was to evaluate the plasma concentration of diclofenac sodium (DS) in dogs submitted to diclofenaco phonophoresis and to evaluate if phonophoresis induces greater absorption of this drug in dogs. Five dogs were used in eight different groups at different times: One group received oral administration of 40mg of DS per dog and seven groups received topical application of emulgel DS. The topical application area was $20 \mathrm{~cm}^{2}$. A continuous ultrasound frequency of $1 \mathrm{MHz}$ and intensity of $0.4 \mathrm{~W} \mathrm{~cm}^{-2}$ was used. Blood collections were performed before the treatment (TO), and $1 \mathrm{~h}(\mathrm{Tl})$ and $4 \mathrm{~h}$ (T2) after ultrasound application for all groups. DS concentrations in plasma were measured by high performance liquid choramatohraphy (HPLC). There was significant increase of DS plasma concentration only at $T 1$ in the oral administration group. It was not possible to detect any concentration of DS in the plasma of dogs after topical application of DS, even after DS phonophoresis. The facilitation of transdermal penetration by ultrasound has not been verified under the protocol specified in this research.
\end{abstract}

Keywords: dog, sonophoresis, diclofenac sodium, HPLC

Recebido em 4 de agosto de 2011

Aceito em 20 de julho de 2012

E-mail: charlespelizzari@yahoo.com.br 


\section{INTRODUÇÃO}

Ultrassom significa "além do som". Trata-se de vibrações mecânicas, produzidas eletricamente (Low e Reed, 2001), inaudíveis, de alta frequência, que podem produzir efeitos fisiológicos térmicos e não térmicos (Prentice, 2003). Na prática médica, essa modalidade terapêutica é uma das mais utilizadas, principalmente com a intenção de estimular a cicatrização e aliviar a dor (Drapper e Prentice, 2004). Quando um feixe de onda ultrassônica atinge uma interface acústica, parte da energia é refletida ou refratada. Quando o ultrassom entra em contato com o ar, produz uma refração quase total da energia. Assim, é necessário meio de acoplamento na interface transdutor e local da aplicação (pele) para permitir que as ondas passem do transdutor para os tecidos (Starkey, 2001).

A movimentação do transdutor durante a aplicação do ultrassom pode reduzir os efeitos danosos das ondas estacionárias. Essa movimentação do transdutor deve ser a uma velocidade aproximada de $4 \mathrm{~cm} \mathrm{~s}^{-1}$ (Drapper e Prentice, 2004). As ondas emitidas podem ser em modo contínuo, em que a energia do ultrassom estará sendo produzida em tempo integral, ou modo pulsado, em que a energia é interrompida em intervalos chamados off time (Drapper e Prentice, 2004).

A administração tópica de medicamentos objetiva a penetração do princípio ativo nos tecidos, tais como músculo e articulações, abaixo do sítio de aplicação. Um problema associado à eficácia do uso tópico dos anti-inflamatórios não esteroidais (AINES) é o acesso ao tecido-alvo: se o princípio ativo penetra nos tecidos mais profundos por difusão simples, a partir do local de aplicação, ou se o fármaco é absorvido pela circulação sanguínea local e, subsequentemente, distribuído pela circulação sistêmica (Solignac, 2004; Petersen e Rovati, 2009).

$\mathrm{Na}$ tentativa de mostrar a eficácia da disponibilidade aumentada do fármaco, foi realizada administração tópica e intravenosa in vivo do piroxicam em ratos. Os resultados obtidos dão suporte à hipótese de que a administração tópica pode levar ao aumento na concentração local do fármaco nos tecidos subjacentes, tal como o músculo, sem antes entrar na circulação geral (McNeill et al., 1992). É importante ressaltar que, em caninos e felinos, a vascularização da região epidérmica da pele é menos desenvolvida do que em seres humanos, antropoides e suínos (Pavletic, 1998).

A utilização local de AINES como forma terapêutica no alívio de afecções inflamatórias e dolorosas tem despertado o interesse de muitos profissionais da saúde e pesquisadores, pois apresenta inúmeras vantagens em relação ao uso sistêmico, principalmente por evitar os efeitos indesejáveis gastrintestinais (Rosim, 2003; Fusaro, 2006; Barnes, 2008).

Existem várias formas de administração medicamentosa. Uma delas é a fonoforese. Fonoforese ou sonoforese é o movimento de fármacos para dentro dos tecidos cutâneos, sob a influência da energia ultrassônica (Low e Reed, 2001; Starkey, 2001). Para Drapper e Prentice (2004), é uma técnica que melhora o fornecimento de medicação específica aos tecidos e que tem como maior vantagem o fato de fornecer medicamento de maneira segura, indolor e não invasiva. Possui ainda o benefício de permitir a penetração nos tecidos, de forma não invasiva, e o de diminuir $o$ efeito de passagem (Starkey, 2001) ou biotransformação, que seria a transformação química de substâncias, pois muitos agentes biotransformados podem ser responsáveis por efeitos tóxicos ao organismo (Florio, 1999). Além disso, a ausência de degradação pelo trato digestório evita efeitos colaterais associados ao metabolismo gastrintestinal, normalmente decorrentes da administração por via oral (Wu et al., 1998). Segundo Fusaro (2006), a via transdérmica associada ao ultrassom terapêutico representa otimização no método, pois ela demonstrou que a fonoforese induziu à maior absorção de diclofenaco dietilamônio.

A aplicação do ultrassom necessita de agentes de acoplamento, que podem ser géis (Starkey, 2001). Em fonoforese, é muito importante a eficácia dos meios de acoplamento, pois alguns deles impedem a passagem do som, podendo-se aplicar separadamente o gel e a medicação, sendo esse método conhecido por "método invisível" (Starkey, 2001). A eficácia da fonoforese não foi totalmente comprovada $\mathrm{e}$ ainda existem controvérsias, muitas delas relacionadas ao tipo de agente acoplador 
utilizado e à concentração do medicamento (Starkey, 2001). Barnes (2008), em estudo sobre a exposição do diclofenaco sódico ao ultrassom, sugere a ausência da degradação do fármaco, elegendo-o para o uso em fonoforese

O diclofenaco sódico é um potente antiinflamatório não esteroidal e analgésico, que tem ação sobre a inibição da ciclooxigenase e da lipoxigenase. Sua utilização em cães é contraindicada devido aos efeitos colaterais, principalmente sangramento gástrico (Tasaka, 1999). É rapidamente absorvido, depois da administração oral, e sua concentração plasmática é atingida dentro de duas a três horas. Estudos em analgesia pós-cesariana, em humanos, indicam que tanto a necessidade de opioides quanto os escores de dor podem ser diminuídos quando o diclofenaco for administrado em diferentes doses e por diferentes vias (Cardoso, 2002). As propriedades analgésicas do diclofenaco têm sido atribuídas aos seus efeitos sobre a síntese periférica de prostaglandinas. $\mathrm{O}$ diclofenaco pode ser administrado por via oral, retal ou parenteral em humanos. A via menos invasiva deve ser sempre a escolhida (Cardoso, 2002).

Em humanos sadios, Porta (1992) administrou diclofenaco sódico peroral, e Rosim (2003)


$225 \mathrm{~cm}^{2}$, tempo de cinco minutos de irradiação, intensidade de $0,5 \mathrm{Wcm}^{-2}$, frequência de $1 \mathrm{Mhz}$ e modo contínuo; ambos os autores constataram facilitação transcutânea. Fusaro (2006), ao administrar diclofenaco dietilamônio, em área de $150 \mathrm{~cm}^{2}$, e adotar o restante do protocolo igual ao de Rosim (2003), observou maior absorção do fármaco por fonoforese, mas $75 \%$ das amostras ficaram abaixo do limite de quantificação, que foi de $50 \mathrm{ngmL}^{-1}$. Todos esses autores avaliaram a concentração plasmática de diclofenaco por cromatografia líquida de alta eficiência (CLAE), mostrando que é um método eficiente de quantificação deste fármaco em plasma sanguíneo.

Assim, o objetivo desta pesquisa foi avaliar a concentração plasmática de diclofenaco sódico em cães após sua aplicação na forma de emulgel por fonoforese sob diferentes protocolos de tratamentos e verificar se a fonoforese interfere na absorção deste fármaco.

\section{MATERIAL E MÉTODO}

A pesquisa seguiu as normas de experimentação, segundo o Comitê de Ética e Bem- Estar Animal da Universidade Federal de Santa Maria, e foi aprovada sob o processo administrativo número 23081.018091/2008-48. Foram utilizados cinco cães, sem raça definida, fêmeas, com massa corporal entre 9 e $16 \mathrm{~kg}$. Antes da experimentação, os animais foram submetidos previamente ao procedimento de ovariossalpingo-histerectomia, sendo alojados em canis individuais por 15 dias para adaptação ao local. Nesse período, os animais receberam alimentação duas vezes ao dia e água à vontade. Foi também administrado anti-helmíntico (pamoato de pirantel/praziquantel), na dose de $25 \mathrm{mg} \mathrm{kg}^{-1}$, e realizaram-se hemograma e avaliação sérica de alanina-aminotransferase (ALT) e fosfatase alcalina (FA). Foram selecionados apenas cães cujos resultados dos exames bioquímicos estivessem dentro da normalidade para a espécie. Após o término do estudo, os animais foram doados.

Foram compostos oito grupos (Fig. 1), e todos os cães participaram de todos eles. Os procedimentos foram: grupo 1 - aplicação de ultrassom acoplado a gel comum para ultrassonografia por seis minutos, remoção do gel com papel-toalha e, após aplicação de $2 \mathrm{~g}$ de diclofenaco sódico (DS) emulgel (1\%), com permanência por seis minutos, retirada do DS; grupo 2 - aplicação de $2 \mathrm{~g}$ de DS emulgel tópico, com permanência por seis minutos, e posterior retirada do DS; grupo 3 - aplicação de $2 \mathrm{~g}$ de DS emulgel, posteriormente recoberto com gel comum para acoplamento e emprego do ultrassom pelo tempo de seis minutos, seguida de retirada do gel comum e do DS; grupo 4 repetição do protocolo do grupo 1, com o ultrassom desligado; grupo 5 - repetição do protocolo do grupo 3, com o ultrassom desligado; grupo 6 - aplicação de $2 \mathrm{~g}$ de DS emulgel tópico e aplicação do ultrassom diretamente sobre o DS pelo tempo de seis minutos, e posteriormente retirada do emulgel excedente; grupo 7 - repetição do protocolo do grupo 6, com o ultrassom desligado; grupo 8 administração oral única de $40 \mathrm{mg}$ de DS comprimido por animal. 


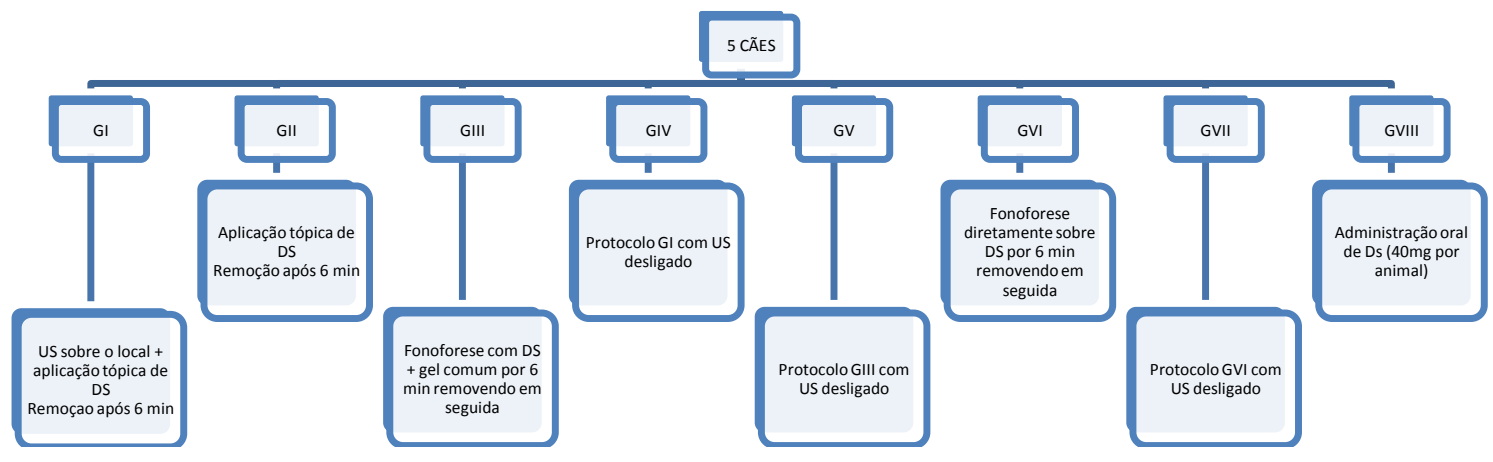

Figura 1. Diagrama representando o protocolo dos grupos de cães submetidos ou não à fonoforese. (DS) = diclofenaco sódico.

Em cada cão, aplicou-se o diclofenaco sódico emulgel no membro pélvico direito, na região lateral da coxa, com movimentos circulares, na velocidade de $4 \mathrm{~cm}^{2} \mathrm{~s}^{-1}$, conforme descrito por Drapper e Prentice (2004). Usou-se área de $20 \mathrm{~cm}^{2}(5 \times 4 \mathrm{~cm})$, demarcada por pincel de tinta, precedida de tricotomia com máquina específica e lâmina número 40, e fez-se a limpeza local com solução fisiológica e álcool 92,8gL. A frequência do ultrassom foi de $1 \mathrm{MHz}$, a área de radiação efetiva do ultrassom foi de $3,5 \mathrm{~cm}^{2}$, modo contínuo, com intensidade de $0,4 \mathrm{Wcm}^{-2}$ pelo tempo de seis minutos, e o meio de acoplamento do cabeçote gel comum ou diretamente diclofenaco sódico conforme protocolo dos grupos. A concentração do gel de diclofenaco sódico foi de $1 \%$ em cada aplicação. A aplicação do US (ultrassom) foi realizada todas as vezes pelo mesmo indivíduo. Cada protocolo foi empregado uma vez em cada animal, e estes foram submetidos ao novo protocolo após o período de sete dias.

Para a avaliação plasmática da concentração de diclofenaco sódico, realizou-se a coleta de amostra de sangue da veia jugular antes de se executarem os protocolos (tempo zero), após uma hora (tempo 1) e após quatro horas da aplicação (tempo 2) em todos os cães de cada grupo. Os animais permaneceram sob restrição alimentar até a última coleta e usaram colar elisabetano para proteção da área de aplicação. Cada amostra foi identificada, colocada em tubos contendo EDTA e, após centrifugação a 3500 x g por 10 minutos, o plasma, extraído com pipeta, foi acondicionado em tubos Eppendorf, identificado e armazenado em freezer até a data da realização da análise por CLAE.

A quantificação do diclofenaco nas amostras de plasma foi realizada utilizando-se equipamento cromatográfico HPLC (CLAE) Waters Alliance ${ }^{\circledR}$ , equipado com detector Waters $2487 \mathrm{UV}$-vis operando no comprimento de onda de $276 \mathrm{~nm}$. A separação foi feita em uma coluna Symmetry ${ }^{\circledR}$ $\mathrm{C} 18$, $5 \mu \mathrm{m}$ de $250 \mathrm{x} 4,6 \mathrm{~mm}$, protegida por précoluna Symmetry ${ }^{\circledR} \mathrm{C} 18,5 \mu \mathrm{m}$ de 3,9x20mm, em fase reversa, composta por isopropanol, água, acetonitrila e tampão acetato $\mathrm{pH} 4,0$, na proporção de $48,22,10$ e $20 \%$, respectivamente, com fluxo de $0,45 \mathrm{~mL} \mathrm{~min}^{-1}$. O processamento foi executado com base no método publicado por

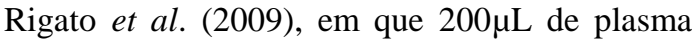
foram tratados com $200 \mu \mathrm{L}$ de padrão interno (naproxeno) em acetonitrila para uma concentração final de $5 \mu \mathrm{g} \mathrm{mL}^{-1}$. A mistura foi agitada em vórtex e, em seguida, centrifugada a $15000 \mathrm{x} \mathrm{g}$ por $10 \mathrm{~min}$ à temperatura de $4{ }^{\circ} \mathrm{C}$. Transferiu-se o sobrenadante para insert, e $100 \mu \mathrm{L}$ dele foram injetados no sistema cromatográfico.

Para análise estatística, aplicou-se análise de variância de uma via, seguida de teste de Tukey $(\mathrm{P}<0,05)$. 


\section{RESULTADOS E DISCUSSÃO}

Somente na administração oral - grupo 8 puderam-se observar concentrações plasmáticas de diclofenaco sódico após uma e quatro horas. No tempo anterior à administração (zero), nenhuma amostra apresentou o produto. Nos outros grupos - 1 a 7 -, não se observou valor plasmático de diclofenaco sódico, ou este estava abaixo do limite de quantificação pelo método bioanalítico desenvolvido, que foi de $25 \mathrm{ng} \mathrm{mL}^{-1}$. Para esses níveis abaixo do limite de quantificação, atribuiu-se valor zero para cálculo estatístico. Houve diferença $(\mathrm{P}<0,05)$ apenas no tempo de uma hora do grupo 8 em relação aos demais tempos e grupos (Fig. 2).



Figura 2. Representação nas variações das concentrações plasmáticas do diclofenaco sódico nos tempos zero, 1 (uma hora) e 2 (quatro horas) em cada cão, nas amostras obtidas a partir do protocolo 8 (via oral) e a mediana. No tempo zero, as amostras não apresentaram valores de diclofenaco quantificáveis $\left(<25 \mathrm{ng} \mathrm{mL}^{-1}\right)$; notar significância $(*)$ no t1.

Este resultado mostra que o método bioanalítico desenvolvido foi adequado para a detecção e a quantificação do diclofenaco em amostras de plasma e que a aplicação do diclofenaco emulgel nos protocolos dos grupos 1 a 7 não determinou absorção para quantificação plasmática do produto.
A utilização do DS foi em razão de sua ação antiinflamatória e analgésica, descrita por Cardoso (2002), e mesmo que este fármaco seja contraindicado para cães (Tasaka, 1999), optouse por utilizá-lo em razão de seu baixo custo e da facilidade de obtenção, e, também, por se acreditar que a via tópica não causaria efeitos colaterais em cães. A dose administrada foi adaptada de Hosny et al. (1996), que utilizaram 
50mg de diclofenaco sódico por cão na raça Beagle com dispositivo retal. Pelo fato de a via de administração ser diferente neste trabalho, foram utilizados $40 \mathrm{mg}$ de diclofenaco sódico por animal. A quantidade de diclofenaco emulgel e o protocolo de aplicação do ultrassom foram adaptados de Rosim (2003), É importante citar que, conforme Barnes (2008), não há um consenso de protocolo padrão concernente à aplicação do ultrassom. A escolha do protocolo, em cães, é basicamente empírica, provavelmente devido ao fato de haver limitado número de publicações na área e de os dados obtidos serem conflitantes ou adaptados de literatura humana.

A área no membro pélvico foi escolhida em razão da facilidade de aplicação e da alta incidência de lesões traumáticas nessa região. A baixa intensidade e o modo pulsado, utilizados nesta pesquisa, são as características do equipamento desenvolvido, o qual tem sido empregado em investigações experimentais e clínicas por Xavier e Duarte (1983) e Rubin et al. (2001). A quantidade de $2 \mathrm{~g}$ de diclofenaco sódico aplicada, nos grupos sem o gel comum para ultrassonografia, foi suficiente para a realização da sessão, evitando que o cabeçote do ultrassom ficasse sem o meio de acoplamento, segundo recomenda Starkey (2001). Os tempos das coletas foram baseados no Dicionário de Especialidades Farmacêuticas Interativo (Dicionário..., 2005/2006), no qual consta que a meia-vida terminal do diclofenaco no plasma é de 1-3 horas.

Não houve diferença $(\mathrm{P}<0,05)$ entre os cães dos grupos submetidos ao ultrassom (fonoforese) e aqueles sem o uso do ultrassom, não se evidenciando valor plasmático de diclofenaco sódico, ou este estava abaixo do limite de quantificação do método bioanalítico desenvolvido. Em estudo com diclofenaco dietilamônio em humanos sadios, Fusaro (2006) relatou que, em $75 \%$ dos voluntários submetidos à fonoforese ou à aplicação tópica sem ultrassom, as concentrações plasmáticas de diclofenaco ficaram abaixo do limite de detecção, que foi de 50ng $\mathrm{mL}^{-1}$. Rosim (2003) mostrou que o ultrassom com diclofenaco sódico facilitou a penetração transcutânea em humanos. Para McNeill et al. (1992) e Fusaro (2006), a quantificação de diclofenaco plasmático pode não ocorrer, mas houve indícios de que possa existir concentração local, abaixo da pele, na área de fonoforese. Carnio (2005), em avaliação da fonoforese com diclofenaco sódico em gelatina imitando pele humana, verificou que a penetração do medicamento foi de $3 \mathrm{~mm}$ para a irradiação com $1,0 \mathrm{Wcm}^{-2}$ e de $5 \mathrm{~mm}$ para a irradiação com $1,5 \mathrm{Wcm}^{-2}$ de forma contínua e estacionária por cinco minutos, confirmando a concentração local. Para testar essa hipótese no cão, seria necessário avaliar a região da área de aplicação, a fim de determinar a presença do DS.

Uma das causas da não quantificação pode estar relacionada à área e ao tempo de exposição do fármaco (McNeill et al., 1992). A área e o tempo de exposição utilizado por Rosim (2003) foram maiores. Ainda, pode-se levar em consideração as propriedades físicas e anatômicas da pele; em caninos e felinos, a vascularização da região epidérmica da pele é menos desenvolvida do que em seres humanos, antropoides e suínos (Pavletic, 1998). Por este motivo, pode-se inferir que haja menor absorção do fármaco em canino. A não quantificação sérica e o entendimento da presença do fármaco na região de aplicação são desejáveis, pois a espécie canina é muito susceptível aos efeitos colaterais do diclofenaco em concentrações plasmáticas elevadas, sendo o ideal que se mantivesse apenas a concentração inibitória mínima local. Portanto, há necessidade de estudos subsequentes em pacientes enfermos para verificar se essa técnica é eficiente no controle anti-inflamatório ou analgésico local.

\section{CONCLUSÕES}

A aplicação tópica de diclofenaco sódico, em cães submetidos ou não à fonoforese, com intensidade de $0,4 \mathrm{Wcm}^{-2}$, modo contínuo, frequência de $1 \mathrm{MHz}$ em tempo de irradiação de seis minutos, não detecta concentração plasmática do fármaco por HPLC, que foi quantificado apenas pela administração oral. O protocolo utilizado demonstra que não há facilitação da penetração transdérmica pelo ultrassom, pela avaliação do plasma por cromatografia líquida de alta eficiência.

\section{AGRADECIMENTO}

Ao $\mathrm{CNPq}$, pelo financiamento do projeto, processo n. 300885/2008-3. 


\section{REFERÊNCIAS}

BARNES, D. Efeito do ultrassom sobre a nocicepção $e$ o processo inflamatório em modelos animais $e$ sobre a estrutura química de fármacos. 2008. 66f. Dissertação (Mestrado em Ambiente e Desenvolvimento) - Centro Universitário Univates, RS.

CARDOSO, M.M.S.C.; CARVALHO, J.C.A.; TAHAMTANI, S.M.M. et al. Diclofenaco por via muscular ou retal associado com baixas doses de morfina subaracnoidea para analgesia pós-operatória em cesarianas. Rev. Bras. Anestesiol., v.52, p.666-672, 2002.

CARNIO, P.B. Variação dos parâmetros físicos do campo ultrassonico em fonoforese com diclofenaco gel. 2005. 77f. Dissertação (Mestrado em Bioengenharia) - Universidade de São Paulo, São Paulo, SP.

DICIONÁRIO de Especialidades Farmacêuticas Interativo. Rio de Janeiro: Publicações Científicas, 2005/06. p.1944

DRAPPER, D.O.; PRENTICE, W.E. Ultrassom terapêutico. In: PRENTICE, W.E. Modalidades terapêuticas para fisioterapeutas. Porto Alegre: Artmed, 2004. Cap.10, p.245-274.

FLORIO, J.C. Absorção, distribuição, biotransformação e eliminação. In: SPINOSA, H.S. et al. Farmacologia aplicada à medicina veterinária. Rio de Janeiro: Guanabara Koogan, 1999. Cap.4, p.34.

FUSARO, C. Estudo da fonoforese de diclofenaco dietilamônio em voluntários sadios. 2006. 53f. Dissertação (Mestrado em Farmacologia) Universidade Estadual de Campinas, SP.

HOSNY, E.A.; NIAZY, E.M.; EL-DARDARI, M.M. Effect of polycarbophil concentration on diclofenac sodium bioavailability from suppositories in beagle dogs. Int. J. Pharm, v.136, p.37-41, 1996.

LOW, J.; REED, A. Ultrassom terapêutico. In: Eletroterapia explicada princípios e prática. São Paulo: Manole, 2001. Cap.06, p.187-228.

McNEILL, S.C.; POTTS, R.O.; FRANCOEUR, M.L. Local enhanced topical delivery (LETD) of drugs: does it truly exist? Pharm Res, v.9, p.1422-1427, 1992.

MITCHELL, J.A.; WARNER, T.D. Cyclo-oxygenase2: pharmacology, physiology, biochemistry andrelevance to NSAID therapy. Br. J. Pharmacol., v.128, p.1121-1132, 1999.
PAVLETIC, M.M. Pele e órgãos anexos. In: SLATER, D. Manual de cirurgia de pequenos animais. São Paulo: Manole, 1998. Cap.24, p.328-329.

PETERSEN, B.; ROVATI, S. Diclofenacepolamine (Flector) patch: evidence for topical activity. Clin. Drug Investig., v.29, p.1-9, 2009.

PORTA, V. Estudo de interação entre a ranitidina e o diclofenaco em voluntários sadios após administração peroral de voltaren 50. 1992. 209f. Dissertação (Mestrado em Ciências Farmacêuticas) - Universidade de São Paulo, São Paulo, SP.

PRENTICE, W.E. Emprego das modalidades terapêuticas de reabilitação. In: PRENTICE, W.E.; VOIGHT, M.L. Técnicas em reabilitação musculoesqueléticas. Porto Alegre: Artmed, 2003. Cap.18, p.275-276.

RIGATO, H.M.; MORENO, R.A.; ORPINELLI, E.Z. et al. A simple high-performance liquid chromatographic method for the determination of diclofenac in human plasma: application to a comparative bioavailability study. Int. J. Clin. Pharmacol. Ther., p.132-140, 2009.

ROSIM, G.C. Análise da influência do ultrassom terapêutico na penetração transcutânea de diclofenco sódico em humanos sadios. 2003. 62f. Dissertação (Mestrado em Bioengenharia) - Universidade de São Paulo, São Paulo SP.

RUBIN, C.; BOLANDER, M.; RYABY, J.P. et al. The uses of low intensity ultrasound to accelerate the helaing of fractures. J.B.S.J., v.83, p.259-270, 2001.

SOLIGNAC, M. Assessment of a topical NSAIDs in the treatment of pain and inflammation. The example of Flector Plaster, a local bioadhesive plaster containing diclofenacepolamine. Pres. Med., v.33, p.10-13, 2004.

STARKEY, C. Ultra-som. In: _. Recursos terapêuticos em fisioterapia. São Paulo: Manole, 2001. Cap. 6, p.277-313.

TASAKA, A.C. Antiinflamatórios não esteroidais. In: SPINOSA, H.S. et al. Farmacologia aplicada a medicina veterinária. Rio de Janeiro: Guanabara Koogan, 1999. Cap.21, p.220.

WU, J.; CHAPPELOW, J.; YANG, J. et al. Defects generated in human stratum corneum specimens by ultrasound. Ultrasound Med. Biol., v.24, p.705-710, 1998.

XAVIER, C.A.M.; DUARTE, L.R. Estimulação ultrasônica do calo ósseo. Rev. Bras. Ortop., v.18, p.73-80, 1983. 